\title{
Gray Matter Morphological Alteration in Obsessive Compulsive Disorder: Evidence for an Inhibitory Control and Emotional R....
}

Article · July 2016

CITATIONS

0

6 authors, including:

\section{Oscar F Goncalves}

University of Minho

183 PUBLICATIONS 1,273 CITATIONS

SEE PROFILE

\section{Angel Carracedo}

University of Santiago de Compostela

1,023 PUBLICATIONS 23,439 CITATIONS

SEE PROFILE
READS

96

Jorge Leite

Harvard Medical School

51 PUBLICATIONS 358 CITATIONS

SEE PROFILE

Some of the authors of this publication are also working on these related projects: 


\section{Principles and Practice of Clinical Research}

A Global Journal in Clinical Research

\title{
Review
}

\section{Gray Matter Morphological Alteration in Obsessive Compulsive Disorder: Evidence for an Inhibitory Control and Emotional Regulation Disorder}

Óscar F. Gonçalves ${ }^{1,2,3^{*}}$, Sandra Carvalho1, ${ }^{2}$, Jorge Leite $1,{ }^{2}$, Ana Fernandes-Gonçalves ${ }^{4}$, Angel Carracedo ${ }^{5}$, Adriana Sampaio ${ }^{1}$

1 *-Corresponding author - Neuropsychophysiology Lab, School of Psychology University of Minho, Campus de Gualtar 4710-057 Braga, Portugal Tel: +351 253604 261; Fax: +351 253604 658; Email: goncalves@psi.uminho.pt.

Received May $19^{\text {th }}$; Revised June $27^{\text {th }}, 2016$; Accepted July $5^{\text {th }}, 2016$

\begin{abstract}
The integration of obsessive compulsive disorders (OCD) in the Obsessive Compulsive and Related Disorders cluster, while emphasizing the centrality of inhibitory control and repetitive behaviors may fail to fully acknowledge the existence of significant affective and other cognitive impairments. The objective of this paper is to present examples on available gray matter imaging studies and meta-analyses that may help understanding cognitive and emotional related factors implicated in OCD. Building on these studies, OCD seems to be a disorder of both inhibitory control and emotional regulation. Volumetric and shape abnormalities in different brain territories of the prefrontal cortex, orbitofrontal cortex, thalamus, striate and cerebellum may contribute to impairments in inhibitory control. On the other side, gray matter shape and volume alterations in regions such as the anterior cingulate, insula, amygdala and supramarginal gyrus may contribute to difficulties in emotional regulation. Beyond this inhibitory control - emotional regulation dichotomy, there are other psychological impairments that may be associated with gray matter alterations. For example, difficulties in memory monitoring may be sustained by shape and volumetric alterations in the dorso-lateral prefrontal cortex and the hippocampus. Additionally, visual-spatial impairments may be explained by gray matter shape and volume alterations in the superior parietal and occipital lobes as well as the precuneus. Overall the research confirms changes in volume and shape in multiple cortical and subcortical regions that can help explaining the complexity of OCD symptomatology and the diversity of OCD endophenotypes. Additionally, the evidence from brain shape anomalies may suggest the additional possibility of neurodevelopmental changes associated with the pathogenesis of OCD.
\end{abstract}

Key-Words: Neuroimaging; Obsessive Compulsive Disorder; Brain Volume; Brain Shape; Inhibitory Control; Emotional Regulation.

$\overline{\text { Citation: Gonçalves OF, Carvalho S, Leite J, Fernandes-Gonçalves A, Carracedo A, Sampaio A. Gray Matter Morphological Alteration in }}$ Obsessive Compulsive Disorder. PPCR 2016, Jan-Apr;1(1):162-171.

Copyright: $\odot 2016$ Gonçalves et al. The Principles and Practice of Clinical Research is an open-access article distributed under the terms of the Creative Commons Attribution License, which allows unrestricted use, distribution, and reproduction in any medium, providing the credits of the original author and source.

\section{Introduction}

Obsessive compulsive is a complex disorder characterized by a diversity of symptoms that involve both affective (i.e., intense emotional arousal associated with intrusive thoughts of impending danger) and cognitive impairments (i.e., difficulties in inhibitory control). While emphasized the affective core by classifying OCD among anxiety disorders, the new DSM-5 (American Psychiatric Association, 2013) underscores the cognitive core, by integrating $\mathrm{OCD}$ in a new diagnostic cluster characterized by impairments in inhibitory control. 
While initial brain imaging research has brought evidence for impairments in inhibitory control pathways, more recent methods are suggesting that multiple brain alterations may be associated with emotional and other cognitive impairments in OCD. Therefore, the objective of the current paper is to provide research evidence from gray matter morphology imaging studies identifying brain abnormalities in OCD that may help understanding cognitive and emotional factors implicated in OCD. Rather than providing another exhaustive review (c.f., Piras et al., 2015), here we intend to build upon available research studies and meta-analyses in order to show that OCD may be better conceptualized as a disorder of both cognitive and emotional regulation. In the context of the present article, cognitive regulation refers to the presence of executive (i.e., inhibitory control, working memory, and cognitive flexibility), memory, and visual-spatial deficits. Likewise, the concept of emotional regulation encompasses the emotional component present in obsessions (i.e., anxiety-evoking obsessions) and the motivational component present in compulsions (i.e., anxiety reduction compulsion). We start by presenting examples of initial findings on volumetric alterations of subcortical structures in OCD followed by discussing abnormalities in additional regions of the cortico-striatalthalamus-cortical (CSTC) pathways. Then, evidence for volumetric abnormalities beyond the subcortical regions and CSTC pathways are presented. These extended volumetric alterations are then discussed with examples of studies with a diversity of OCD endophenotypes. Finally, examples of results from shape alterations in gray matter of OCD patients are presented.

\section{Initial Findings on Volumetric Alteration of Subcortical Structures}

The initial neuroanatomic studies conducted with OCD patients showed contradictory evidence of volumetric changes in subcortical structures, particularly in the caudate nucleus. The caudate nucleus is a major basal ganglia structure and part of frontal-striatal pathways involved in cognitive, emotional and motor regulation (Hélie, Ell, \& Ashby, 2015). However, these early findings failed to be consistent across studies. For example, Scarone et al. (1992) carried out a study comparing the volume of the caudate nucleus head in patients diagnosed with OCD when compared with normal controls. Significant volume enlargement of the head of the right caudate nucleus was found in OCD, independently of the patients' clinical and demographic characteristics. Contrasting with these early reports on volumetric increase of the caudate nuclei, a study Robinson et al. (1995) comparing OCD with healthy controls, found a decreased volume of the caudate nucleus independently of the severity or duration of OCD symptoms. Curiously, Aylward et al. (1996), using structural MRI failed to show volumetry differences between OCD and controls of both, the caudate and putamen nuclei.

\section{Volumetric alteration of cortico-striatal- thalamus-cortical pathways}

Since these early contradictory findings, several authors have reported volumetric changes in other cortical and subcortical structures that are part of the CSTC pathways. CSTC pathways project from the cortex to the striatum, to the thalamus (via the globus pallidus) and back to the cortex. A discussed previously, distinct CSTC loops may be involved in distinct regulatory processes (Milad \& Rauch, 2012).

Volumetric alterations in regions associated with CSTC pathways have been consistently reported in OCD, namely: globus pallidus, thalamus, anterior cingulate and orbitofrontal cortex. For example, Szeszko et al. (2004) researched volumetric changes of the caudate nucleus, putamen, globus pallidus, anterior cingulate and superior frontal gyrus in non-medicated children diagnosed with OCD when compared with healthy controls. The authors found a significant volumetric decrease in the globus pallidus along with nan increase in the anterior cingulate in the OCD sample. Interestingly, no significant differences were found for the caudate, putamen and superior frontal gyrus volumes.

As discussed above, the orbitofrontal cortex is part of CSTC pathways involved in inhibitory control, a function that constitutes a core impairment in OCD (J. Fan et al., 2016). The orbitofrontal cortex was reported altered in several studies with OCD patients. For example, changes in orbitofrontal cortex volumes were analyzed in an early study by Choi et al. (2004) comparing OCD patients with a sample of normal controls. As expected, a decrease in the volume of the left anterior region of the orbitofrontal cortex was found in OCD patients. Interestingly, a recent study on the effects of cognitive behavioral therapy observed that patients with OCD exhibited volume increases in the orbitofrontal after effective treatment (Atmaca et al., 2016).

Several quantitative meta-analyses confirmed OCD brain volumetric changes in regions associated with the distinct CSTC loops. For example, Rotge et al. (2009) conducted the first quantitative meta-analysis of MRI studies looking for brain structural abnormalities in OCD patients. In this analysis, 14 non voxel-based morphometry studies were included. The following brain regions were considered: whole brain, intracranial volume and gray matter volume, prefrontal cortex, left and right anterior cingulate cortex, left and right orbitofrontal 
cortex, left and right thalamus volumes, left and right putamen, total, left, and right caudate nucleus. The results of this meta-analysis showed a significantly volumetric decrease in the left anterior cingulate and bilateral orbitofrontal cortices as well as a reliable increase of the thalamus in OCD patients. Finally, meta-regression analyses showed that the effects-sizes on thalamus volume were partially predicted by symptom severity as measured by the Yale-Brown Obsessive-Compulsive Scale (YBOCS). As mentioned before, the thalamus is a core relay structure in all the CSTC loops described above and may play an integrative role between these segregated pathways accomplishing a mutual regulations between motor, cognitive and affective components (Haber \& Calzavara, 2009). Moreover, decrease of the anterior cingulate volume may be associated with alterations of the emotional regulatory pathways projecting from the anterior cingulate and ventromedial prefrontal cortex to the accumbens, thalamus and back to the cortex (Gianaros \& Wager, 2015).

The existence of alterations of CSTC pathways illustrated by volumetric abnormalities in the left anterior cingulate, orbitofrontal cortex (i.e., reduced volume) and the thalamus (i.e., increased volume) reported in this first meta- analysis, was further explored in a study carried out by Rotge, Dilharreguy, et al. (2009) in which the relationship between thalamus and orbitofrontal and anterior cingulated volumes was analyzed in a sample of OCD patients and healthy controls. As anticipated, in OCD patients, but not in the healthy controls, orbitofrontal volume was negatively correlated with the thalamus volume. However, no significant relationship was found between thalamic and anterior cingulate volumes in either OCD patients and controls. Similar findings were reported in review of studies with pediatric populations, showing evidence for CSTC pathways abnormalities (Huyser, Veltman, de Haan, \& Boer, 2009).

In a second meta-analysis, Radua and Mataix-Cols (2009) analyzed twelve studies comparing OCD and healthy controls using whole-brain voxel based morphometry. OCD patients were found to have significant volume increases in gray matter in the lenticular nucleus (particularly ventral anterior putamen but with extensions also to the caudate), confirming abnormalities in subcortical regions associated with two distinct CSTC loops: dorsolateral-caudate-thalamus-cortex and orbitofrontal-putamen-thalamus-cortex. On the contrary, OCD patients exhibited significant decreases of gray matter in dorsal medial frontal/anterior cingulate gyri, with extensions to the supplemental motor area and frontal eye fields, affecting still a third CSTC loop (anterior cingulateaccumbens-thalamus-cortex). Finally, and most interestingly, the authors also observed a significant increase in right superior parietal lobe gray matter, particularly evident in patients without comorbid depression. This is indeed the first meta-analysis confirming morphological changes in areas outside these pathways, namely the right superior parietal lobe, a brain region that has been traditionally associated with attention and visual-spatial processing (Wang et al., 2015).

Summing up, extensive research shows the existence of gray matter volumetric alterations in CSTC territories associated with regulation of motor, cognitive and emotional processes. More recently, new research evidence suggests more extensive structural volumetric abnormalities in regions outside these pathways. We will turn now to discussing examples of this evidence.

\section{Volumetric Abnormalities beyond the CSTC Loops}

The finding of morphometric abnormalities in regions outside the CSTC loops was further confirmed in another meta-analysis of voxel-based morphometry studies carried out by Rotge et al. (2010). Included in this meta-analysis were ten studies using voxel-based morphometry comparing OCD with healthy controls. Consistent with previous analysis, the authors confirmed gray matter alterations in orbitofrontal-basal ganglia pathways in OCD patients (e.g., basal ganglia, lateral orbitofrontal cortex, superior frontal gyrus, dorsolateral prefrontal cortex, and medial frontal gyrus). Again, and most interesting, significant smaller gray matter was found in a region of the parietal cortex - the supramarginal gyrus. This gyrus is thought to be involved in complex visual processing tasks (i.e., reading) as well as mental simulation of anticipated events (Schacter, Addis, \& Buckner, 2007). This finding of volumetric alterations in the supramarginal gyrus may contribute to the overestimation of negative consequences of anticipated events frequently found in OCD patients (Exner, Zetsche, Lincoln, \& Rief, 2014).

Using a different method (signed differential mapping meta-analysis), Peng et al. (2012) looked at alterations of gray and white matter in voxel based and diffusion studies with OCD. The authors confirmed reduced gray matter in several frontal regions (frontal eye fields, medial frontal gyrus, anterior cingulate cortex) along with increases in several subcortical nuclei (lenticular, caudate). Again, and consistently with Radua and Mataix-Cols (2009) review, a region of the superior parietal lobe was found also to have increased gray matter volume.

Atmaca (2011), in a qualitative of review of structural neuroimaging studies, confirmed the involvement of orbitofrontal cortex and thalamus regions but point also that volumetric alterations of hippocampus-amygdala regions are particularly present in refractory obsessivecompulsive disorder. These alterations of the amygdalahippocampus complex may be associated with 
impairments in emotional and memory processing. In fact, functional studies reported abnormal activations of the amygdala in OCD patients, particularly in the case of aggression/checking and sexual/religious symptoms (Via et al., 2014). Volumetric alterations in the hippocampus may contribute to impairments in the processing of contextual cues in memory tasks (Olson et al., 2016).

Two more recent meta-analyses confirmed some of same gray matter alterations in OCD presented above. In what was probably the largest multicenter voxel based morphometry mega-analysis ever carried out, de Wit et al. (2014) looked at both gray and white matter volumes in OCD patients $(n=412)$ and healthy controls $(n=368)$ taking advantage of the "International OCD Brain Imaging Consortium" databases. The authors found significant reductions in gray matter in OCD patients in several regions of the frontal cortex (i.e., inferior frontal cortex and anterior insula; dorsomedial prefrontal cortex and anterior cingulate). An increased gray matter volume was observed on the cerebellum of OCD patients. The authors interpreted this last finding in terms of the role of the cerebellum in cognitive and emotional regulatory processes and its functional connection with CSTC pathways. Alteration in the cerebellum can thus be seen as additional contributions to the prevalence of selfregulatory impairments in OCD.

Another recent review of voxel-based morphometry studies by Piras et al. (2015) confirms widespread gray matter changes in OCD. Eighteen studies were reviewed with a total of 511 OCD patients and 504 healthy controls. The authors point out that, besides the typical volumetric alterations described in studies and reviews reported above, there is a progressive emergence of volumetric reductions in other regions such as dorsal medial frontal cortex along with morphological alterations in the temporal, parietal and occipital regions.

All together several volumetric abnormalities were found in regions within and outside the CSTC loops which may contribute to the impairments of several psychological functions in OCD, namely: (1) volumetric abnormalities in the thalamus, orbitofrontal cortex, striate and cerebellum which may play an important role in executive and inhibitory control impairments; (2) volume alterations in the anterior cingulate, supramarginal gyrus and the amygdala may contribute to difficulties in emotional regulation; (3) volume changes in the hippocampus and the dorsolateral cortex are potentially associated with memory alterations; (4) finally, volume abnormalities the superior parietal and occipital lobes may contribute to deficits in visual-spatial processing.

\section{Gray matter volumetric alterations and symptom dimensions in OCD}

Despite the wide consensus on the alteration of the frontal-subcortical structures, the data remains somehow inconsistent across the different studies. Part of these inconsistencies may be due to the different segmentation techniques used (Despotović et al., 2015). However, another possibility is that the inconsistencies may be due to the symptomatic heterogeneity present in OCD (Markarian et al., 2010). In other words, the question is to know if the different subtypes of OCD (e.g., contamination; harming; symmetry/ordering; pure obsessions; hoarding) are associated with specific structural alterations.

Pujol et al. (2004) did one of the first studies trying to map different structural changes in different OCD subtypes. Using a voxel-based morphometry approach, the authors compared the differences in gray matter density in OCD when compared with healthy controls. OCD patients were assessed in five symptomatic dimensions: symmetry/ordering, hoarding, contamination/cleaning, aggressive/checking, and sexual/religious. Consistent with the reviews reported above, the authors found reductions in prefrontal structures (medial frontal, orbitofrontal, and left inferior insulo-opercular), along with increases in the subcortical regions (ventral striatum) and the anterior cerebellum. However, no overall differences in the different regions described above were associated with the five symptomatic dimensions. However, OCD patients with higher scores on the aggressive/checking dimension had significant volumetric reductions in the right amygdala.

Building on these early findings, a subsequent voxelbased morphometry study by Gilbert et al. (2008) compared gray matter volume in OCD patients and matched healthy controls, using, this time, the ObsessiveCompulsive Inventory - Revised (Abramowitz \& Deacon, 2006), classifying patients in one of the following symptomatic conditions: washing, checking, hoarding, and ordering. As in previous studies, there were significant gray matter reductions in prefrontal regions (e.g., BA9, BA6, BA46, and BA8). For the first time volumetric increases in midbrain structures (which may help sustain alterations of the monoaminergic systems) were shown. It is important to note that these changes were significantly associated with the degree of symptom severity. More important for the current discussion, some symptomatic dimensions were related with different neuroanatomic changes. More specifically, patients with prominent washing symptoms showed significant reductions in the posterior right frontal lobe. Additionally, a slight association was reported between hoarding and the posterior left frontal lobe. As pointed out by the authors, these posterior frontal regions are mostly involved in complex motor coordination and may help explain the 
repetitive compulsive behavior so characteristic in both washers and hoarders.

Later, a study by Van Den Heuvel et al. (2009) using voxel-based morphometry, brought new evidence of the neuroanatomical changes associated with different symptomatic dimensions in OCD patients. In this study, medication-free OCD patients were compared with agematched healthy controls in terms of global a regional gray and white matter volumetry. Additionally, the study analyzed the relationship between neuroanatomic structural changes and OCD symptomatic dimensions (i.e., contamination/washing; harm/checking; symmetry/ordering). The authors found that, in spite of the absence of significant differences between OCD and controls in terms of overall gray and white matter volumes, OCD patients presented decreased regional gray matter in several frontal regions (left lateral orbitofrontal, left inferior frontal cortex, left dorsolateral prefrontal cortex, bilateral medial prefrontal cortex) along with white matter changes (bilateral prefrontal lobes). For the purpose of the present review we will restrict our discussion to gray matter changes. In the OCD group, the study found that regional gray matter was negatively correlated with the following symptomatic dimensions: contamination/washing (bilateral caudate); harm/checking (bilateral temporal); symmetry/ordering (bilateral parietal). Interesting to note the association found between neuroanatomic changes and symptomatic dimensions, suggesting that the dorsal caudate may be associated with the lack of control over washing compulsions, bilateral anterior temporal lobe atrophy may explain difficulties in memory monitoring common in checking rituals and, finally, parietal cortex impairment may underlie some attentional and visualspatial processes in symmetry/ordering symptoms.

A study by Subirà et al. (2013) explored the association of gray matter volumetric alterations with two distinct categories of OCD patients: autogenous obsessions (i.e., aggressive, sexual or religious obsessions) and reactive obsessions (i.e., contamination, errors, accidents or symmetry). The authors showed that, compared with healthy controls, both groups of patients had decreased volumetry in the middle temporal gyrus (a finding consistent with data from patients with harm/checking OCD symptoms). More interesting however, reactive patients differed from healthy controls by showing increased volumes in bilateral putamen. Contrastingly, the autogenous cohort had a significantly volume decrease in the anterior temporal lobe, when compared with healthy controls. This data may suggest that, while reactive obsessions are more associated with inhibitory control functions, autogenous obsessions may involve impairments in high-level cognition, such as memory monitoring and semantic processing.
The fact that different types of symptomatic expressions in OCD are associated with very specific gray matter alteration is also well illustrated in a recent study by Subirà et al. (2015). The authors showed that patients with sensory phenomenon symptoms (i.e., uncomfortable bodily sensations preceding or accompanying repetitive rituals) had an increased gray matter density in the bilateral medial sensorimotor cortex. This finding is consistent with studies showing hyperexcitability of the sensorimotor cortex in OCD patients (Russo et al., 2014).

Also, a recent study by Segalàs et al. (2014) looked for gray matter changes in OCD patients associated with alterations of olfactory processing (i.e., detection threshold, discrimination, and identification). The study showed that OCD patients had significantly gray matter increases in the left anterior cingulate and left orbitofrontal cortex. Significant positive correlations were found between the volume of the left anterior cingulate and odor detection threshold as well as between volume in the left orbitofrontal cortex and odor identification errors. Interestingly, both of these brain regions are involved in olfactory processing suggesting, once again, the association between symptomatic characteristics and volumetric alteration in OCD.

On another interesting study Alonso et al. (2013) reported initial evidence that the prevalence of dysfunctional beliefs (e.g., perfectionism/intolerance to uncertainty; overimportance/need to thought control) may be explained by volumetric decreases in the anterior temporal lobe. This data is consistent with the volumetric alterations observed in patients with autogenous obsessions.

Summarizing, there is increased evidence that specific volumetric alterations in gray matter in OCD may be symptom specific. The findings reported above, with patients from different OCD subgroups, suggest that alterations in gray matter volume in specific brain regions may sustain characteristic impairments. Starting from the more general to the more specific: (1) reactive obsessions showed increased volumes in putamen (a region associated with inhibitory control functions) while autogenous obsessions are associated with volumetric decreases in the anterior temporal lobe (regions sustaining memory updating and semantic processing); (2) gray matter volumes were reported to be negatively correlated with the contamination/washing (bilateral caudate - lack of inhibitory control); harm/checking (bilateral temporal memory monitoring); symmetry/ordering (bilateral parietal - attentional and visual spatial processes); (3) specific symptoms present in some patients are associated with volumetric gray matter alteration in regions relevant to the type of specific processing required (e.g. increased gray matter in the medial sensorimotor cortex for sensory 
phenomenon symptoms; increased volume of the left anterior cingulate and orbitofrontal cortex associated with impairments in olfactory processing; decreased anterior temporal lobe gray matter associated with predominance of dysfunctional beliefs).

\section{Gray Matter Shape Alterations in $O C D$}

We have been showing data demonstrating the existence of changes in the volume of several neuroanatomic gray matter structures in OCD patients, particularly in the CSTC regions, but also with growing evidence for the involvement of more posterior structures, such as temporal, parietal and even cerebellar regions, along with regions of the medial temporal lobe (e.g. amygdala and hippocampus). The gray matter abnormalities assessed with voxel based morphometry methods can be influenced by shape alterations. Therefore, it may be relevant to look for differences in shape between OCD patients and matched healthy controls. Important to note that shape alterations are consider a reliable indication of abnormal brain development, and may contribute to understand the possible neurodevelopmental roots of OCD.

A good marker for the identification of brain shape difference is the study of anomalies in cortical folding as expressed by the gyrification index (ratio between the inner and outer contours). A study by Wobrock et al. (2010) compared the level of cortical folding (using an automated gyrification index) of the prefrontal cortex in OCD when compared with healthy controls. Several results of this study are worthy of mentioning here. First, a significant hypogyrification was found in the left prefrontal cortex of OCD patients with a tendency for an equivalent hypogyrification on the right prefrontal cortex. Second, and contrasting with healthy controls, no significant correlations were found between volume and gyrification of the prefrontal cortex in OCD patients. Both of these results, even though preliminary, may point that brain shape changes, as assessed by the level of cortical folding of the frontal cortex, may constitute brain markers for OCD.

Alteration of gyrification patterns were also found in a study by Shim, Jung, Choi, and Jung (2009) analyzing the sulcus morphology in the anterior cingulate cortex regions (i.e., the paracingulate and cingulate sulci) in OCD compared with normal controls. The authors found initial evidence for the existence of a less developed paracingulate sulcus in the left hemisphere in OCD patients. Likewise, more recently, Q. Fan et al. (2013) studying gyrification, along with thickness and surface changes, in unmedicated OCD patients and healthy controls, observed that OCD patients had an increase of gyrification in the left insula, left middle frontal and left lateral occipital regions and the parietal regions of the precuneus and right supramarginal gyrus.
Further evidence of frontal-basal ganglia shape abnormalities was reported in a study of (Pujol et al., 2011) using a two-dimensional morphometric approach with a large sample of OCD patients and healthy controls. Comparisons of the distance between different frontal and basal ganglia structures to the anterior commissure (medial frontal; anterior lateral frontal; anterior insula and caudate) revealed deformations in OCD patients of the anterior-posterior dimensions of both frontal and basal ganglia along with a frontal opercula displacement.

The finding of shape abnormalities on selected subcortical structures was also reported in several studies. For example, J. S. Choi et al. (2007) examined shape changes of the three structures of the striatum (caudate nucleus; putamen; globus pallidus) in obsessive compulsive patients when compared with healthy controls. The authors observed that OCD patients exhibited significant shape bilateral outward abnormalities in the superior and anterior portion of the caudate nucleus (more evident on the right side). Additionally, significant outward deformities were also found in the inferior, lateral region of the left putamen. As remarked by the authors, these shape deformities seem to affect areas linked with the cognitive associative function of the striatum (i.e., anterior caudate) as well as the emotional limbic connections (i.e., inferior putamen) and motor executive control (i.e., posterior putamen), that may underlie cognitive, emotional and motor deficits in OCD.

Recently, Kubota et al. (2016) also reported alterations of shape in the putamen region were also. In a study with a group of healthy participants, the authors correlated putamen shape and volume with the Maudsley Obsessive Compulsive Inventory (MOCI). The authors found a significant association between MOCI scores and hypertrophy of the anterior region of bilateral putamen. In another study, Kang et al. (2008) researched alterations in thalamus shape morphology in OCD patients when compared with schizophrenic patients and healthy matched controls. Even though no significant volumetric changes were found for the OCD group, there was evidence that patients with OCD exhibited abnormalities in the shape of the anterior lateral right thalamus and the posterior left thalamus. Additionally, a decreased asymmetry of the pulvinar nucleus region was found in OCD patients. As suggested by the authors, the thalamic shape deformities are probably related with difficulties in behavior inhibition (ventral anterior and ventral lateral thalamus) and attention and working memory (pulvinar nucleus). These changes in the shape of subcortical structures are consistent with the recent findings reported by (Shaw et al., 2015) on surface expansions in striatal and thalamic regions both in OCD and unaffected high genetic risk siblings. 
Consistent with data from volumetric studies, initial evidence for the existence of shape deformities in areas outside the CSTC pathways were also reported in a study by Hong et al. (2007). In this study, the authors analyzed hippocampus shape in OCD patients and matched emotional regulation), supramarginal gyrus (possible association with mental anticipation), and occipital cortex (possible association with visual spatial processing); (2) shape abnormalities in the striatum (anterior caudate possible association with cognitive regulation; inferior

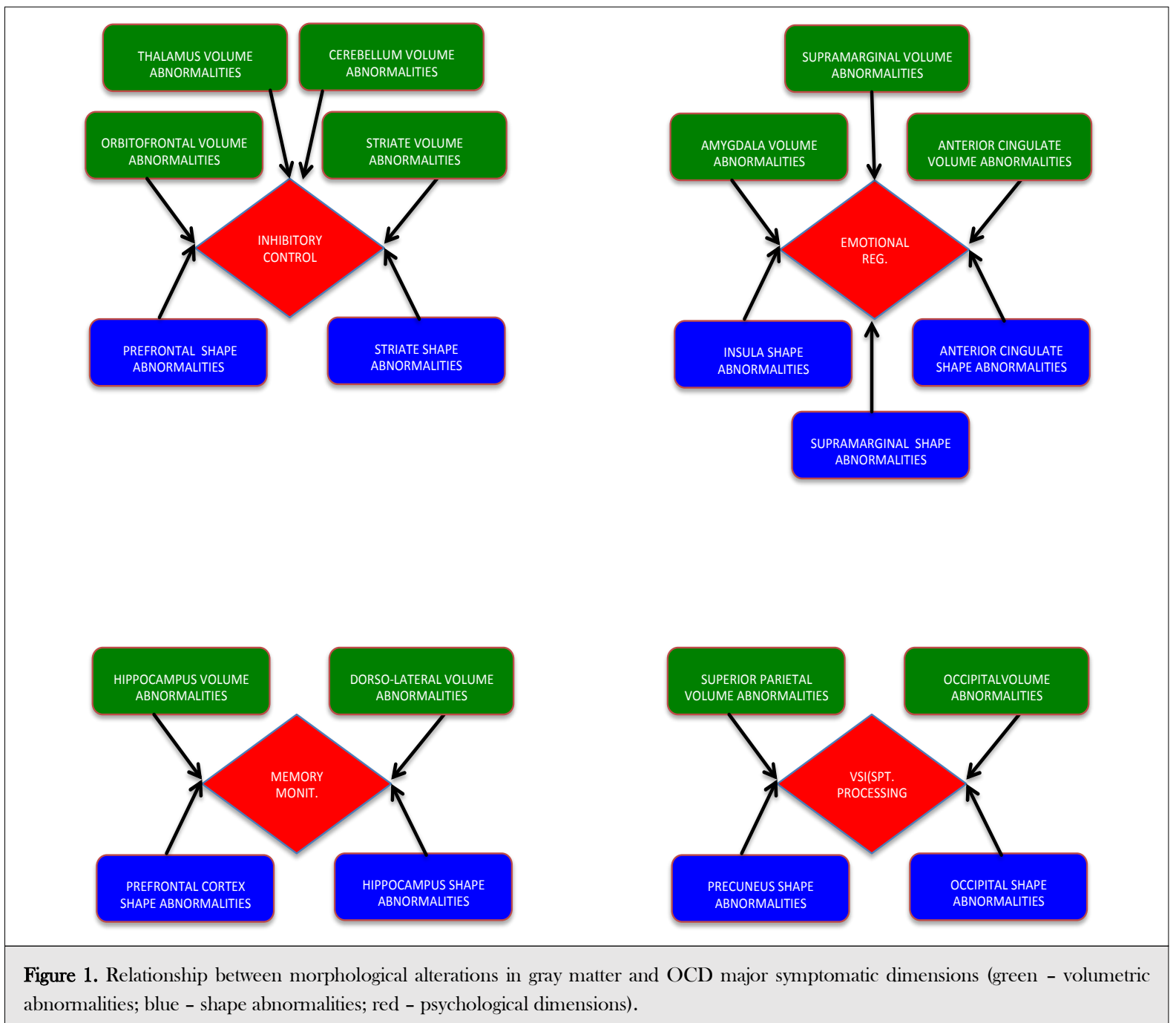

healthy controls and found bilateral deformities in OCD

putamen - possibly associated with emotional regulation;

patients, particularly the anterior portion (superior surface inward deformity and an inferior surface outward deformity). These data, along with the absence of a ignificant positive correlation with volume changes, brings initial evidence that some of neurocognitive findings such as visual-spatial memory impairments may be independent from alterations related to the CSTC network.

Putting together the data from shape analysis in OCD we may conclude that, consistent with volumetric studies, shape alterations were reported in regions within and outside CSTC loops. These results help explaining the complexity and diversity of OCD symptoms, more specifically: (1) alterations of gyrification in the prefrontal cortex (possible association with executive impairments), insula (possible associations with embodied emotional processing), anterior cingulate (possible association with and posterior putamen - possible associations with executive control); thalamic region (ventral anterior and ventral lateral thalamus - possible associations with inhibitory control deficits; pulvinar nucleus - possible associations with attention and working memory impairments), and hippocampus (possible associations with contextual and visual spatial memory).

\section{Conclusion}

The research on gray matter morphological alterations in OCD confirms changes in volume and shape of gray matter in areas associated with the CSTC pathways, particularly dorsolateral cortex, orbitofrontal cortex, anterior cingulate, thalamus and striate. However, there is increased evidence for the involvement of regions outside these pathways, such as several regions of the parietal 
cortex as well as amygdala, hippocampus and cerebellum. The neuroanatomical involvement of multiple cortical and subcortical regions can help to explain not only the complexity of OCD symptomatology but also the diversity of OCD endophenotypes. Data on volumetric and shape changes in different OCD endophenotypes is not systematically documented, so it would be important for future studies to evaluate specific alterations according to type of OCD symptoms and also patients' clinical features. Additionally, the evidence from brain shape anomalies, while confirming data from volumetric studies, suggests the additional possibility of neurodevelopmental changes associated with the pathogenesis of OCD.

Previous critical reviews have discussed the relationship between cognitive functioning and OCD gray matter alterations (Menzies et al., 2008). Here we show that these gray matter abnormalities are present in brain regions associated with a diversity of cognitive and emotional processes. The findings discussed above are consistent with a recent review showing that compulsivity, as illustrated in OCD, is associated with structural abnormalities in brain networks sustaining emotional, cognitive, and motor functioning (Van den Heuvel et al., 2016).

Figure 1 tries to capture the association between volumetric and shape alterations and some OCD psychological features, listing the most obvious connections (green - volumetric abnormalities; blue shape abnormalities; red - psychological dimensions). Volumetric and shape abnormalities in different brain territories of the prefrontal cortex, orbitofrontal cortex, thalamus, striate and cerebellum may contribute to impairments in inhibitory control. On the other side, gray matter shape and volume alterations in regions such as the anterior cingulate, insula, amygdala and supramarginal gyrus may sustain difficulties in emotional regulation. Beyond this inhibitory control - emotional regulation dichotomy, there are other psychological impairments that may be explained by gray matter alterations reported here. For example, difficulties in memory monitoring may be associated with shape and volumetric alterations in the dorsolateral prefrontal cortex and the hippocampus respectively. Additionally, visual-spatial impairments often reported in OCD may be, at least partially, explained by gray matter shape and volume alterations in the superior parietal and occipital lobe as well as the precuneus.

Needless to say that not infrequently, the same brain region may be involved in several psychological dimensions. For example, the cerebellum seems to contribute both to inhibitory control and emotional regulation, while, for instance to supramarginal gyrus or the precuneus play important roles in both visual spatial processing and emotional regulation. Additionally, there is now abundant functional evidence for the interaction between different brain networks in OCD (Göttlich, Krämer, Kordon, Hohagen, \& Zurowski, 2014).

Concluding, and building on gray matter evidence, OCD seems to be a disorder of both cognitive and emotional regulation. This conclusion is consistent with evidence from white matter (Koch, Reess, Rus, Zimmer, \& Zaudig, 2014) and functional studies (Del Casale et al., 2011). While there is now some evidence for the relationship between specific cognitive and emotional impairments and specific OCD clinical features (e.g., memory - compulsive checking), future studies should better clarified the direction of this causality (Radomsky, Dugas, Alcolado, \& Lavoie, 2014). The challenge for future research is to integrate multimodal neuroimaging methods in order to provide an integrated model for the multiplicity of psychological impairments in OCD. A final comment to note that the first line psychotherapeutic treatment for OCD involves two strategies intended to deal with each of those mechanisms present in OCD Exposure and Response/Ritual Prevention. While exposure involves confronting patients with symptom provoking stimuli with the objective of regulating emotional expression, response/ritual prevention is intended to enhance inhibitory control (Arch \& Abramowitz, 2015). The research reported above brings neuroscience support for this approach, while pointing out to the need of attending to other domains beyond inhibit control (e.g., memory; visual-spatial processing) and fear processing (e.g., disgust, shame). Additionally, evidence of regional gray matter alterations associated with specific OCD symptoms may contribute to better guide the targets for invasive (Alonso et al., 2015) and non invasive brain stimulation (Pedapati et al., 2015) as well as for neuromodulation via real time fMRI (Buyukturkoglu et al., 2015).

\section{Conflict of interest and financial disclosure}

The authors followed the International Committee or Journal of Medical Journals Editors (ICMJE) form for disclosure of potential conflicts of interest. All listed authors concur with the submission of the manuscript, the final version has been approved by all authors. The authors have no financial or personal conflicts of interest.

\section{Acknowledgement}

The first author was funded by the Brazilian National Counsel for Scientific and Technological Development (CNPq) as a Special Visiting Researcher of the Science Without Borders program (grant number: 401143/2014-7). This study was partially conducted at the Neuropsychophysiology Lab from the Psychology Research Centre (UID/PSI/01662/2013), University of Minho, and supported by the Portuguese Foundation for Science and Technology and the Portuguese Ministry of Science, Technology and Higher Education through national funds and co-financed by FEDER through COMPETE2020 under the PT2020 Partnership Agreement (POCI-01-0145- FEDER-007653). This work was also supported by the Portuguese Foundation for Science and Technology (FCT) and European Union (FSE-POPH) with two individual grants (SFRH/BPD/86041/2012 and SFRH/BPD/86027/2012). 


\section{Authors' affiliations}

1 Neuropsychophysiology Lab, CIPsi, School of Psychology, University of Minho, Braga - Portugal.

${ }^{2}$ Spaulding Center of Neuromodulation, Department of Physical Medicine \& Rehabilitation, Spaulding Rehabilitation Hospital and Massachusetts General Hospital, Harvard Medical School, Boston, MA, USA

Department of Applied Psychology, Bouvé College of Health Sciences, Northeastern University, Boston-USA.

${ }^{4}$ CUF Porto Hospital, Department of Psychiatry, Porto - Portugal

Forensic Genetics Unit, Institute of Legal Medicine, Faculty of Medicine, University of Santiago de Compostela, Galicia-Spain

\section{References}

Abramowitz, J. S., \& Deacon, B. J. (2006). Psychometric properties and construct validity of the Obsessive-Compulsive Inventory-Revised: Replication and extension with a clinical sample. Journal of Anxiety Disorders, 20(8),

http://doi.org/10.1016/j.janxdis.2006.03.001

Alonso, P., Cuadras, D., Gabri??ls, L., Denys, D., Goodman, W., Greenberg, B. D., ... Menchon, J. M. (2015). Deep brain stimulation for obsessive-compulsive disorder: A meta-analysis of treatment outcome and predictors of response. PLOS ONE, 1O(7), 1-16. http://doi.org/10.1371/journal.pone.0133591

Alonso, P., Orbegozo, A., Pujol, J., López-Solà, C., Fullana, M. À., Segalàs, C., ... Soriano-Mas, C. (2013). Neural correlates of obsessive-compulsive related dysfunctional beliefs. Progress in Neuro-Psychopharmacology \& Biological Psychiatry, 47, 25-32. http://doi.org/10.1016/j.pnpbp.2013.07.016

American Psychiatric Association. (2000). DSM-IV. Diagnostic and Statistical Manual of Mental Disorders 4th edition TR. Washington, DC: Author

American Psychiatric Association. (2013). DSM-V. Diagnostic and Statistical Manual of Mental Disorders 5th edition. Washington, DC: Author.

Arch, J. J., \& Abramowitz, J. S. (2015). Exposure therapy for obsessivecompulsive disorder: An optimizing inhibitory learning approach. Journal of Obsessive-Compulsive and Related Disorders, 6, 174182. http://doi.org/10.1016/j.jocrd.2014.12.002

Atmaca, M. (2011). Review of structural neuroimaging in patients with refractory obsessivecompulsive disorder. Neuroscience Bulletin, 27(3), 215-220. http://doi.org/10.1007/s12264-011-1001-(

Atmaca, M., Yildirim, H., Yilmaz, S., Caglar, N., Mermi, O., Korkmaz, S., ... Turkcapar, H. (2016). Orbito-frontal cortex and thalamus volumes in the patients with obsessive-compulsive disorder before and after cognitive behavioral therapy. International Journal of Psychiatry in Medicine, 0091217415621038 http://doi.org/10.1177/0091217415621038

Aylward, E. H., Harris, G. J., Hoehn-Saric, R., Barta, P. E., Machlin, S. R., \& Pearlson, G. D. (1996). Normal caudate nucleus in obsessivecompulsive disorder assessed by quantitative neuroimaging. Archives of General Psychiatry, 53(7), 577-84.

Buyukturkoglu, K., Roettgers, H., Sommer, J., Rana, M., Dietzsch, L., Arikan, E. B., ... Ruiz, S. (2015). Self-Regulation of Anterior Insula with Real-Time fMRI and Its Behavioral Effects in ObsessiveCompulsive Disorder: A Feasibility Study. PloS One, 10(8), e0135872. http://doi.org/10.1371/journal.pone.0135872

Choi, J. S., Kim, S. H., Yoo, S. Y., Kang, D. H., Kim, C. W., Lee, J. M., ... Kwon, J. S. (2007). Shape deformity of the corpus striatum in obsessive-compulsive disorder. Psychiatry Research - Neuroimaging, 155(3), 257-264. http://doi.org/10.1016/j.pscychresns.2007.02.004

Choi, J.-S. S., Kang, D.-H. H., Kim, J.-J. J., Ha, T.-H. H., Lee, J.-M. M., Youn, T., ... Kwon, J.-S. S. (2004). Left anterior subregion of orbitofrontal cortex volume reduction and impaired organizational strategies in obsessive-compulsive disorder. Journal of Psychiatric Research, $38(2)$, http://doi.org/10.1016/j.jpsychires.2003.08.001

de Wit, S. J., Alonso, P., Schweren, L., Mataix-Cols, D., Lochner, C., Menchón, J. M., ... van den Heuvel, O. A. (2014). Multicenter voxel-based morphometry mega-analysis of structural brain scans in obsessive-compulsive disorder. The American Journal of Psychiatry, 171(3), 340-349. http://doi.org/10.1176/appi.ajp.2013.13040574

Del Casale, A., Kotzalidis, G. D., Rapinesi, C., Serata, D., Ambrosi, E., Simonetti, A., ... Girardi, P. (2011). Functional neuroimaging in obsessive-compulsive disorder. Neuropsychobiology, 64(2), 61-85. http://doi.org/10.1159/000325223

Despotović, I., Goossens, B., Philips, W., T, I. D., Goossens, B., \& Philips, W. (2015). MRI Segmentation of the Human Brain: Challenges, Methods, and Applications. Computational and Mathematical Methods in Medicine, 2015, 450341. http://doi.org/http://dx.doi.org/10.1155/2015/450341

Exner, C., Zetsche, U., Lincoln, T. M., \& Rief, W. (2014). Imminent danger? Probabilistic classification learning of threat-related information in obsessive-compulsive disorder. Behavior Therapy, 45(2), 157-167. http://doi.org/10.1016/j.beth.2013.09.006

Fan, J., Liu, W., Lei, H., Cai, L., Zhong, M., Dong, J., ... Zhu, X. (2016). Components of inhibition in autogenous- and reactive-type obsessive-compulsive disorder: Dissociation of interference control. Biological Psychology, 117, http://doi.org/10.1016/j.biopsycho.2016.03.008

Fan, Q., Palaniyappan, L., Tan, L., Wang, J., Wang, X., Li, C., ... Liddle, P. F. (2013). Surface anatomical profile of the cerebral cortex in obsessive-compulsive disorder: a study of cortical thickness, folding and surface area. Psychological Medicine, 43(5), 1081-1091. http://doi.org/10.1017/S0033291712001845

Gianaros, P. J., \& Wager, T. D. (2015). Brain-Body Pathways Linking Psychological Stress and Physical Health. Current Directions in $\begin{array}{llr}\text { Psychological Science, } & \text { 24(4), }\end{array}$ http://doi.org/10.1177/0963721415581476

Gilbert, A. R., Mataix-Cols, D., Almeida, J. R. C., Lawrence, N., Nutche, J., Diwadkar, V., ... Phillips, M. L. (2008). Brain structure and symptom dimension relationships in obsessive-compulsive disorder: A voxel-based morphometry study. Journal of Affective Disorders, 109(1-2), 117-126. http://doi.org/10.1016/j.jad.2007.12.223

Göttlich, M., Krämer, U. M., Kordon, A., Hohagen, F., \& Zurowski, B. (2014). Decreased limbic and increased fronto-parietal connectivity in unmedicated patients with obsessive-compulsive disorder. Human Brain Mapping, $\quad 35(11), \quad 5617-5632$. http://doi.org/10.1002/hbm.22574

Haber, S. N., \& Calzavara, R. (2009). The cortico-basal ganglia integrative network: the role of the thalamus. Brain Research Bulletin, 78(2-3), 69-74. http://doi.org/10.1016/j.brainresbull.2008.09.013

Hélie, S., Ell, S. W., \& Ashby, F. G. (2015). Learning robust corticocortical associations with the basal ganglia: an integrative review. Cortex, 64, 123-35. http://doi.org/10.1016/j.cortex.2014.10.011

Hong, S. B., Shin, Y. W., Kim, S. H., Yoo, S. Y., Lee, J. M., Kim, I. Y., ... Kwon, J. S. (2007). Hippocampal shape deformity analysis in obsessive-compulsive disorder. European Archives of Psychiatry and Clinical Neuroscience, 257(4), 185-190. http://doi.org/10.1007/s00406-006-0655-5

Huyser, C., Veltman, D. J., de Haan, E., \& Boer, F. (2009). Paediatric obsessive-compulsive disorder, a neurodevelopmental disorder?. Evidence from neuroimaging. Neuroscience and Biobehavioral Reviews, 33(6), 818-830. http://doi.org/10.1016/j.neubiorev.2009.01.003

Kang, D.-H., Kim, S. H. I., Kim, C.-W., Choi, J.-S., Jang, J. H., Jung, M. H., ... Kwon, J. S. (2008). Thalamus surface shape deformity in obsessive-compulsive disorder and schizophrenia. Neuroreport, 19(6), 609-613. http://doi.org/10.1097/WNR.0b013e3282fa6db9

Koch, K., Reess, T. J., Rus, O. G., Zimmer, C., \& Zaudig, M. (2014). Diffusion tensor imaging (DTI) studies in patients with obsessivecompulsive disorder (OCD): a review. Journal of Psychiatric Research, 54, 26-35. http://doi.org/10.1016/j.jpsychires.2014.03.006

Kubota, Y., Sato, W., Kochivama, T., Uono, S., Yoshimura, S., Sawada, R., ... Toichi, M. (2016). Putamen volume correlates with obsessive compulsive characteristics in healthy population. Psychiatry Research. http://doi.org/10.1016/j.pscychresns.2016.01.014

Markarian, Y., Larson, M. J., Aldea, M. A., Baldwin, S. A., Good, D., Berkeljon, A., ... McKay, D. (2010). Multiple pathways to functional impairment in obsessive-compulsive disorder. Clinical Psychology Review, 30(1), 78-88. http://doi.org/10.1016/j.cpr.2009.09.005

Menzies, L., Chamberlain, S. R., Laird, A. R., Thelen, S. M., Sahakian, B. J., \& Bullmore, E. T. (2008). Integrating evidence from neuroimaging and neuropsychological studies of obsessivecompulsive disorder: The orbitofronto-striatal model revisited. Neuroscience and Biobehavioral Reviews, 32(3), 525-549. http://doi.org/10.1016/j.neubiorev.2007.09.005

Milad, M. R., \& Rauch, S. L. (2012). Obsessive-compulsive disorder: beyond segregated cortico-striatal pathways. Trends in Cognitive Sciences, 16(1), 43-51. http://doi.org/10.1016/j.tics.2011.11.003 
Olson, C. A., Hale, L. R., Hamilton, N., Powell, J. N., Martin, L. E., \& Savage, C. R. (2016). Altered source memory retrieval is associated with pathological doubt in obsessive-compulsive disorder. Behavioural Brain Research, 296, 53-60. http://doi.org/10.1016/j.bbr.2015.08.031

Pedapati, E., DiFrancesco, M., Wu, S., Giovanetti, C., Nash, T., Mantovani, A., ... Harris, E. (2015). Neural correlates associated with symptom provocation in pediatric obsessive compulsive disorder after a single session of sham-controlled repetitive transcranial magnetic stimulation. Psychiatry Research $\begin{array}{llr}\text { Neuroimaging, } & \text { 233(3), }\end{array}$ http://doi.org/10.1016/j.pscychresns.2015.07.020

Peng, Z.-W., Lui, S. S. Y., Cheung, E. F. C., Jin, Z., Miao, G. D., Jing, J., \& Chan, R. C. K. (2012). Brain structural abnormalities in obsessivecompulsive disorder: Converging evidence from white matter and grey matter. Asian Journal of Psychiatry, 5(4), 290-296. http://doi.org/10.1016/j.ajp.2012.07.004

Piras, F., Piras, F., Chiapponi, C., Girardi, P., Caltagirone, C., \& Spalletta, G. (2015). Widespread structural brain changes in OCD: a systematic review of voxel-based morphometry studies. Cortex, 62, 89-108. http://doi.org/10.1016/j.cortex.2013.01.016

Pujol, J., Soriano-Mas, C., Alonso, P., Cardoner, N., Menchón, J. M., Deus, J., \& Vallejo, J. (2004). Mapping structural brain alterations in obsessive-compulsive disorder. Archives of General Psychiatry, 61(7), 720-730. http://doi.org/10.1001/archpsvc.61.7.720

Pujol, J., Soriano-Mas, C., Gispert, J. D., Bossa, M., Reig, S., Ortiz, H., ... Olmos, S. (2011). Variations in the shape of the frontobasal brain region in obsessive-compulsive disorder. Human Brain Mapping; 32(7), 1100-1108. http://doi.org/10.1002/hbm.21094

Radomsky, A. S., Dugas, M. J., Alcolado, G. M., \& Lavoie, S. L. (2014). When more is less: Doubt, repetition, memory, metamemory, and compulsive checking in OCD. Behaviour Research and Therapy, 59, 30-39. http://doi.org/10.1016/j.brat.2014.05.008

Radua, J., \& Mataix-Cols, D. (2009). Voxel-wise meta-analysis of grey matter changes in obsessive-compulsive disorder. British Journal of Psychiatry, 195(5),

393-402. http://doi.org/10.1192/bjp.bp.108.055046

Robinson, D., Wu, H., Munne, R. A., Ashtari, M., Alvir, J. M., Lerner, G., ... Bogerts, B. (1995). Reduced caudate nucleus volume in obsessive-compulsive disorder. Archives of General Psychiatry, 52(5), 393-398.

Rotge, J. Y., Langbour, N., Jaafari, N., Guehl, D., Bioulac, B., Aouizerate, B., ... Burbaud, P. (2010). Anatomical Alterations and Symptom-Related Functional Activity in Obsessive-Compulsive Disorder Are Correlated in the Lateral Orbitofrontal Cortex. Biological Psychiatry, 67(7), 37-38. http://doi.org/10.1016/j.biopsych.2009.10.007

Rotge, J.-Y., Dilharreguy, B., Aouizerate, B., Martin-Guehl, C., Guehl, D., Jaafari, N., ... Burbaud, P. (2009). Inverse relationship between thalamic and orbitofrontal volumes in obsessive-compulsive disorder. Progress in Neuro-Psychopharmacology \& Biological Psychiatry, 33(4),

682-7. http://doi.org/10.1016/j.pnpbp.2009.03.011

Rotge, J.-Y., Guehl, D., Dilharreguy, B., Tignol, J., Bioulac, B., Allard, M., ... Aouizerate, B. (2009). Meta-analysis of brain volume changes in obsessive-compulsive disorder. Biological Psychiatry, 65(1), 7583. http://doi.org/10.1016/j.biopsych.2008.06.019

Russo, M., Naro, A., Mastroeni, C., Morgante, F., Terranova, C., Muscatello, M. R., ... Quartarone, A. (2014). Obsessive-compulsive disorder: A "sensory-motor" problem? International Journal of Psychophysiology, $92(2)$ http://doi.org/10.1016/j.ijpsycho.2014.02.007

Scarone, S., Colombo, C., Livian, S., Abbruzzese, M., Ronchi, P., Locatelli, M., ... Smeraldi, E. (1992). Increased right caudate nucleus size in obsessive-compulsive disorder: detection with magnetic resonance imaging. Psychiatry Research, 45(2), 115-21.

Schacter, D. L., Addis, D. R., \& Buckner, R. L. (2007). Remembering the past to imagine the future: the prospective brain. Nature Reviews. $\quad$ Neuroscience, $\quad 8(9), \quad 657-661$. http://doi.org/10.1038/nrn2213

Segalàs, C., Alonso, P., Orbegozo, A., Real, E., Subirà, M., López-Solà, C., ... Soriano-Mas, C. (2014). Brain structural imaging correlates of olfactory dysfunction in obsessive-compulsive disorder. European Archives of Psychiatry and Clinical Neuroscience, 264(3), 225-233. http://doi.org/10.1007/s00406-013-0439-7

Shaw, P., Sharp, W., Sudre, G., Wharton, A., Greenstein, D., Raznahan, A., ... Rapoport, J. (2015). Subcortical and cortical morphologica anomalies as an endophenotype in obsessive-compulsive disorder
Molecular Psychiatry, 20(2), 224-231. http://doi.org/10.1038/mp.2014.3

Shim, G., Jung, W. H., Choi, J., \& Jung, M. H. (2009). Reduced cortical folding of the anterior cingulate cortex in obsessive - compulsive disorder. Journal of Psychiatry \& Neuroscience, 34(6), 443-449.

Subirà, M., Alonso, P., Segalàs, C., Real, E., López-Solà, C., Pujol, J., ... Soriano-Mas, C. (2013). Brain structural alterations in obsessivecompulsive disorder patients with autogenous and reactive obsessions. PloS One, 8(9), e75273. http://doi.org/10.1371/journal.pone.0075273

Subirà, M., Sato, J. R., Alonso, P., do Rosário, M. C., Segalàs, C., Batistuzzo, M. C., ... Soriano-Mas, C. (2015). Brain structural correlates of sensory phenomena in patients with obsessivecompulsive disorder. Journal of Psychiatry \& Neuroscience, 40(4), 232-240.

Szeszko, P. R., MacMillan, S., McMeniman, M., Chen, S., Baribault, K., Lim, K. O., ... Rosenberg, D. R. (2004). Brain structural abnormalities in psychotropic drug-naive pediatric patients with obsessive-compulsive disorder. The American Journal of Psychiatry, 161(6), 1049-56. http://doi.org/15169693

Van Den Heuvel, O. A., Remijnse, P. L., Mataix-Cols, D., Vrenken, H., Groenewegen, H. J., Uylings, H. B. M., ... Veltman, D. J. (2009). The major symptom dimensions of obsessive-compulsive disorder are mediated by partially distinct neural systems. Brain, 132(4), 853868. http://doi.org/10.1093/brain/awn267

Van den Heuvel, O. A., van Wingen, G., Soriano-Mas, C., Alonso, P., Chamberlain, S. R., Nakamae, T., ... Veltman, D. J. (2016). Brain Circuitry of Compulsivity. European Neuropsychopharmacology (In Press), 1-18. http://doi.org/10.1016/j.euroneuro.2015.12.005

Via, E., Cardoner, N., Pujol, J., Alonso, P., López-Solà, M., Real, E., ... Harrison, B. J. (2014). Amygdala activation and symptom dimensions in obsessive-compulsive disorder. The British Journal of Psvchiatry, 204(1), 61-68. http://doi.org/10.1192/bjp.bp.112.123364

Wang, J., Yang, Y., Fan, L., Xu, J., Li, C., Liu, Y., ... Jiang, T. (2015). Convergent functional architecture of the superior parietal lobule unraveled with multimodal neuroimaging approaches. Human Brain Mapping, 36(1), 238-57. http://doi.org/10.1002/hbm.22626

Wobrock, T., Gruber, O., McIntosh, A. M., Kraft, S., Klinghardt, A., Scherk, H., ... Moorhead, T. W. (2010). Reduced prefrontal gyrification in obsessive-compulsive disorder. European Archives of Psychiatry and Clinical Neuroscience, 260(6), 455-464. http://doi.org/10.1007/s00406-009-0096-z. 